\title{
Locality of the mean curvature of rectifiable varifolds
}

\author{
Gian Paolo Leonardi and Simon Masnou \\ Communicated by N. Fusco
}

\begin{abstract}
The aim of this paper is to investigate whether, given two rectifiable $k$-varifolds in $\mathbb{R}^{n}$ with locally bounded first variations and integer-valued multiplicities, their mean curvatures coincide $\mathscr{H}^{k}$-almost everywhere on the intersection of the supports of their weight measures. This so-called locality property, which is well known for classical $C^{2}$ surfaces, is far from being obvious in the context of varifolds. We prove that the locality property holds true for integral 1-varifolds, while for $k$-varifolds, $k>1$, we are able to prove that it is verified under some additional assumptions (local inclusion of the supports and locally constant multiplicities on their intersection). We also discuss a couple of applications in elasticity and computer vision.
\end{abstract}

Keywords. Varifolds, mean curvature, semicontinuity, Willmore energy, image reconstruction.

AMS classification. 49Q15, 49Q20.

\section{Introduction}

Let $M$ be a $k$-dimensional rectifiable subset of $\mathbb{R}^{n}, \theta$ a positive function which is locally summable with respect to $\mathscr{H}^{k}\left\llcorner M\right.$, and $T_{x} M$ the tangent space at $\mathscr{H}^{k}$-almost every $x \in M$. The Radon measure $V=\theta \mathscr{H}^{k}\left\llcorner M \otimes \delta_{T_{x} M}\right.$ on the product space

$$
\mathrm{G}_{k}\left(\mathbb{R}^{n}\right)=\mathbb{R}^{n} \times\left\{k \text {-dim. subspaces of } \mathbb{R}^{n}\right\}
$$

is an example of a rectifiable $k$-varifold.

Varifolds can be loosely described as generalized surfaces endowed with multiplicity ( $\theta$ in the example above) and were initially considered by F. Almgren [2] and W. Allard [1] for studying critical points of the area functional.

Unlike currents, they do not carry information on the positive or negative orientation of tangent planes, hence cancellation phenomena typically occurring with currents do not arise in this context. A weak (variational) concept of mean curvature naturally stems from the definition of the first variation $\delta V$ of a varifold $V$, which represents, as in the smooth case, the initial rate of change of the area with respect to smooth perturbations. This explains why it is often natural, as well as useful, to represent minimizers of area-type functionals as varifolds.

One of the main difficulties when dealing with varifolds is the lack of a boundary operator like the distributional one acting on currents. In several situations, one can 
circumvent this problem by considering varifolds that are associated to currents, or that are limits (in the sense of varifolds) of sequences of currents (see [11, 17, 18]).

This paper focuses on varifolds with locally bounded first variation. In this setting, the mean curvature vector $H_{V}$ of a varifold $V$ is defined as the Radon-Nikodym derivative of the first variation $\delta V$ (which can be seen as a vector-valued Radon measure) with respect to the weight measure $\|V\|$ (see Section 1 for the precise definitions). In the smooth case, i.e. when $V$ represents a smooth $k$-surface $S$ and $\theta$ is constant, $H_{V}$ coincides with the classical mean curvature vector defined on $S$.

However, it is not clear at all whether this generalized mean curvature satisfies the same basic properties of the classical one. In particular, it is well known that if two smooth, $k$-dimensional surfaces have an intersection with positive $\mathscr{H}^{k}$ measure, then their mean curvatures coincide $\mathscr{H}^{k}$-almost everywhere on that intersection. Thus it is reasonable to expect that the same property holds for two integral $k$-varifolds having a non-negligible intersection. The importance of assuming that the varifolds are integral (i.e., with integer-valued multiplicities) is clear, as one can build easy examples of varifolds with smoothly varying multiplicities, such that the corresponding mean curvatures are not even orthogonal to the tangent planes (see also the orthogonality result for the mean curvature of integral varifolds obtained by K. Brakke [9]).

This locality property of the generalized mean curvature is, however, far from being obvious, since varifolds, even the rectifiable ones, need not be regular at all. A famous example due to K. Brakke [9] consists of a varifold with integer-valued multiplicity and bounded mean curvature, that cannot even locally be represented as a union of graphs.

Previous contributions to the locality problem are the papers [4] and [18]. In [4], the locality is proved for integral $(n-1)$-varifolds in $\mathbb{R}^{n}$ with mean curvature in $\mathrm{L}^{p}$, where $p>n-1$ and $p \geq 2$. The result is strongly based on a quadratic tilt-excess decay lemma due to R. Schätzle [17]. Taking two varifolds that locally coincide and whose mean curvatures satisfy the integrability condition above, the locality property is proved in [4] via the following steps:

(i) calculate the difference between the two mean curvatures in terms of the local behavior of the tangent spaces;

(ii) remove all points where both varifolds have same tangent space;

(iii) finally, show that the rest goes to zero in density, thanks to the decay lemma [17].

The limitation to the case of varifolds of codimension 1, whose mean curvature is in $\mathrm{L}^{p}$ with $p>n-1, p \geq 2$, is not inherent to the locality problem itself, but rather to the techniques used in R. Schätzle's paper [17] for proving the decay lemma.

A major improvement has been obtained by R. Schätzle himself in [18]. Indeed, he shows that, in any dimension and codimension, and assuming only the $\mathrm{L}_{\mathrm{loc}}^{2}$ summability of the mean curvature, the quadratic decays of both tilt-excess and height-excess 
are equivalent to the $\mathrm{C}^{2}$-rectifiability of the varifold. Consequently, the locality property is shown to hold for $\mathrm{C}^{2}$-rectifiable $k$-varifolds in $\mathbb{R}^{n}$ with mean curvature in $\mathrm{L}^{2}$, as stated in Corollary 4.2 in [18]: Let $V_{1}, V_{2}$ be integral $k$-varifolds in $U \subset \mathbb{R}^{n}$ open, with $H_{V_{i}} \in \mathrm{L}_{\mathrm{loc}}^{2}\left(\left\|V_{i}\right\|\right)$ for $i=1$, 2. If the intersection of the supports of the varifolds is $C^{2}$-rectifiable, then $H_{V_{1}}=H_{V_{2}}$ for $\mathscr{H}^{k}$-almost every point of the intersection.

A careful inspection of the proof of the locality property in [4] and [18] shows the necessity of controlling only those parts of the varifolds that do not contribute to the weight density, but possibly to the curvature. However, the tilt-excess decay provides a local control of the variation of tangent planes on the whole varifold, which seems to be slightly more than what is actually needed for the locality to hold. This observation has led us to tackle this problem by means of different techniques, in order to weaken the requirement on the integrability of the mean curvature down to $\mathrm{L}_{\mathrm{loc}}^{1}$. Our main results in this direction are:

(i) in the case of two integral 1-varifolds (in any codimension) with locally bounded first variations, we prove that the two generalized curvature vectors coincide $\mathscr{H}^{1}$-almost everywhere on the intersection of the supports (Theorem 2.1);

(ii) in the general case of rectifiable $k$-varifolds, $k>1$, we prove that if $V_{1}=$ $\mathbf{v}\left(M_{1}, \theta_{1}\right), V_{2}=\mathbf{v}\left(M_{2}, \theta_{2}\right)$ are two rectifiable $k$-varifolds with locally bounded first variations, and if there exists an open set $A$ such that $M_{1} \cap A \subset M_{2}$ and both $\theta_{1}, \theta_{2}$ are constant on $M_{1} \cap A$, then the two generalized mean curvatures coincide $\mathscr{H}^{k}$-almost everywhere on $M_{1} \cap A$ (Theorem 3.4).

The strategy of proof consists of writing the total variation in a ball $B$ in terms of a $(k-1)$-dimensional integral over the sphere $\partial B$ and showing that this integral can be well controlled, at least for a suitable sequence of nested spheres whose radii decrease toward zero.

The 1-dimensional result is somehow optimal, as the only required hypothesis is the local boundedness of the first variation. Under this minimal assumption, we can prove that there exists a sequence of nested spheres that meet only the intersection of the two varifolds, i.e. essentially the part that counts for the weight density. In other words, the parts of the varifolds that do not contribute to the weight density do not either intersect these spheres. This is a key argument to prove that the curvature is essentially not altered by the presence of these "bad" parts.

In the general $k$-dimensional case it is no more possible to prove the existence of nested spheres that do not intersect at all the bad parts. But we are able to prove, under the extra assumptions cited above, that the integral over the $(k-1)$-dimensional sections of the bad parts with a suitable sequence of spheres is so small, that it does not contribute to the mean curvature, and thus the locality holds true in this case.

The plan of the paper is as follows: in Section 1 we recall basic notations and main facts about varifolds. Section 2 is devoted to the proof of the locality property for 
integral 1-varifolds in $\mathbb{R}^{n}$ with locally bounded first variation (Theorem 2.1), whose immediate consequence is the fact that for any such varifold, the generalized curvature $\kappa(x)$ coincides with the classical curvature of any $C^{2}$ curve that intersects the support of the varifold, for $\mathscr{H}^{1}$-almost all $x$ in the intersection (Corollary 2.2). We also provide an example of a 1-varifold in $\mathbb{R}^{2}$ whose generalized curvature belongs to $\mathrm{L}^{1} \backslash \bigcup_{p>1} \mathrm{~L}^{p}$. In Section 3 we first derive two useful, local forms of the isoperimetric inequality for varifolds due to W. K. Allard [1]. Then, we prove that for almost every $r>0$, the integral of the mean curvature vector in $B_{r}$ coincides with the integral of a conormal vector field along the sphere $\partial B_{r}$, up to an error due to the singular part of the first variation. These preliminary results are then combined to show that an improved decay of the $(n-1)$-weight of the "bad" parts contained in $\partial B_{r}$ holds true, at least for a suitable sequence of radii $\left(r_{h}\right)_{h}$ converging to 0 . This decay argument is the core of the proof of our locality result for $k$-varifolds in $\mathbb{R}^{n}$ (Theorem 3.4).

Finally, we discuss in Section 4 some applications of the locality property for varifolds, in particular to lower semicontinuity results for the Euler's elastica energy and for Willmore-type functionals that appear in elasticy and in computer vision.

\section{Notations and basic definitions}

Let $\mathbb{R}^{n}$ be equipped with its usual scalar product $\langle\cdot, \cdot\rangle$. Let $\mathrm{G}_{n, k}$ be the Grassmannian of all unoriented $k$-subspaces of $\mathbb{R}^{n}$. We shall often identify in the sequel an unoriented $k$-subspace $S \in \mathrm{G}_{n, k}$ with the orthogonal projection onto $S$, which is represented by the matrix $S^{i j}=\left\langle e_{i}, S\left(e_{j}\right)\right\rangle,\left\{e_{1}, \ldots, e_{n}\right\}$ being the canonical basis of $\mathbb{R}^{n} . \mathrm{G}_{n, k}$ is equipped with the metric

$$
\|S-T\|:=\left(\sum_{i, j=1}^{n}\left(S^{i j}-T^{i j}\right)^{2}\right)^{\frac{1}{2}} .
$$

For an open subset $U \subset \mathbb{R}^{n}$ we define $\mathrm{G}_{k}(U)=U \times \mathrm{G}_{n, k}$, equipped with the product metric.

By a $k$-varifold on $U$ we mean any Radon measure $V$ on $\mathrm{G}_{k}(U)$. Given a varifold $V$ on $U$, a Radon measure $\|V\|$ on $U$ (called the weight of $V$ ) is defined by

$$
\|V\|(A)=V\left(\pi^{-1}(A)\right), \quad A \subset U \text { Borel, }
$$

where $\pi$ is the canonical projection $(x, S) \mapsto x$ of $\mathrm{G}_{k}(U)$ onto $U$. We denote by $\Theta^{k}(\|V\|, x)$ the $k$-dimensional density of the measure $\|V\|$ at $x$, i.e.

$$
\Theta^{k}(\|V\|, x)=\lim _{r \rightarrow 0} \frac{\|V\|\left(B_{r}(x)\right)}{\omega_{k} r^{k}},
$$

$\omega_{k}$ being the standard $k$-volume of the unit ball in $\mathbb{R}^{k}$. Recall that $\Theta^{k}(\|V\|, x)$ is well defined $\|V\|$-almost everywhere $[19,10]$. 
Given $M$, a countably $\left(\mathscr{H}^{k}, k\right)$-rectifiable subset of $\mathbb{R}^{n}[10,3.2 .14]$ (from now on, we shall simply say $k$-rectifiable), and given $\theta$, a positive and locally $\mathscr{H}^{k}$-integrable function on $M$, we define the $k$-rectifiable varifold $V \equiv \mathbf{v}(M, \theta)$ by

$$
V(A)=\int_{\pi(T M \cap A)} \theta d \mathscr{H}^{k}, \quad A \subset G_{n}(U) \text { Borel, }
$$

where $T M=\left\{\left(x, T_{x} M\right): x \in M^{*}\right\}$ and $M^{*}$ stands for the set of all $x \in M$ such that $M$ has an approximate tangent space $T_{x} M$ with respect to $\theta$ at $x$, i.e. for all $f \in \mathrm{C}_{\mathrm{c}}^{0}\left(\mathbb{R}^{n}\right)$,

$$
\lim _{\lambda \downarrow 0} \lambda^{-k} \int_{M} f\left(\lambda^{-1}(z-x)\right) \theta(z) d \mathscr{H}^{k}(z)=\theta(x) \int_{T_{x} M} f(y) d \mathscr{H}^{k}(y) .
$$

Remark that $\mathscr{H}^{k}\left(M \backslash M^{*}\right)=0$ and the approximate tangent spaces of $M$ with respect to two different positive $\mathscr{H}^{k}$-integrable functions $\theta, \tilde{\theta}$ coincide $\mathscr{H}^{k}$-a.e. on $M$ (see [19], 11.5).

Finally, it is straightforward from the definition above that

$$
\|V\|=\theta \mathscr{H}^{k}\llcorner M .
$$

Whenever $\theta$ is integer valued, $V=\mathbf{v}(M, \theta)$ is called an integral varifold.

Before giving the definition of the mean curvature of a varifold, we recall that for a smooth $k$-manifold $M \subset \mathbb{R}^{n}$ with smooth boundary, the following equality holds for any $X \in \mathrm{C}_{\mathrm{c}}^{1}\left(\mathbb{R}^{n}, \mathbb{R}^{n}\right)$ :

$$
\int_{M} \operatorname{div}_{M} X d \mathscr{H}^{k}=-\int_{M}\left\langle H_{M}, X\right\rangle d \mathscr{H}^{k}-\int_{\partial M}\langle\eta, X\rangle d \mathscr{H}^{n-1},
$$

where $H_{M}$ is the mean curvature vector of $M$, and $\eta$ is the inner conormal of $\partial M$, i.e. the unit normal to $\partial M$ which is tangent to $M$ and points into $M$ at each point of $\partial M$. The formula involves the tangential divergence of $X$ at $x \in M$ which is defined by

$$
\operatorname{div}_{M} X(x):=\sum_{i=1}^{n} \nabla_{i}^{M} X_{i}(x)=\sum_{i=1}^{n}\left\langle e_{i}, \nabla^{M} X_{i}(x)\right\rangle=\sum_{j=1}^{k}\left\langle\nabla X(x) \tau_{j}, \tau_{j}\right\rangle
$$

where $\left\{\tau_{1}, \ldots, \tau_{k}\right\}$ is an orthonormal basis for $T_{x} M$, with $\nabla^{M} f(x)=T_{x} M(\nabla f(x))$ being the projection of $\nabla f(x)$ onto $T_{x} M$.

The first variation $\delta V$ of a $k$-varifold $V$ on $U$ is the linear functional on $\mathrm{C}_{\mathrm{c}}^{1}\left(U, \mathbb{R}^{n}\right)$ defined by

$$
\delta V(X):=\int_{\mathrm{G}_{k}(U)} \operatorname{div}_{S} X d V(x, S)
$$


where, for any $S \in \mathrm{G}_{n, k}$, we have set $\nabla^{S} X_{i}=S\left(\nabla X_{i}\right)$ and

$$
\operatorname{div}_{S} X=\sum_{i=1}^{n}\left\langle e_{i}, \nabla^{S} X_{i}\right\rangle .
$$

In the case of a $k$-rectifiable varifold $V, \delta V(X)$ is actually the initial rate of change of the total weight $\|V\|(U)$ under the smooth flow generated by the vector field $X$. More precisely, let $X \in \mathrm{C}_{\mathrm{c}}^{1}\left(U, \mathbb{R}^{n}\right)$ and $\Phi(y, \epsilon) \in \mathbb{R}^{n}$ be defined as the flow generated by $X$, i.e. the unique solution to the Cauchy problem at each $y \in U$

$$
\frac{\partial}{\partial \epsilon} \Phi(y, \epsilon)=X(\Phi(y, \epsilon)), \quad \Phi(y, 0)=y .
$$

Then, one can consider the push-forwarded varifold $V_{\epsilon}=\Phi(\cdot, \epsilon)_{\#} V$, for which one obtains

$$
\left\|V_{\epsilon}\right\|(U)=\int_{U} J_{y}^{M} \Phi(y, \epsilon) d\|V\|(y)=\int_{U}\left|1+\epsilon \operatorname{div}_{M} X(y)+o(\epsilon)\right| d\|V\|(y),
$$

where $J_{y}^{M} \Phi(y, \epsilon)=\left|\operatorname{det}\left(\nabla_{y}^{M} \Phi(y, \epsilon)\right)\right|$ is the tangential Jacobian of $\Phi(\cdot, \epsilon)$ at $y$, and therefore

$$
\delta V(X)=\int_{U} \operatorname{div}_{M} X(y) d\|V\|(y)=\frac{d}{d \epsilon}\left\|V_{\epsilon}\right\|(U)_{\mid \epsilon=0}
$$

(see [19, §9 and §16] for more details).

A varifold $V$ is said to have a locally bounded first variation in $U$ if for each $W \subset \subset$ $U$ there is a constant $c<\infty$ such that $|\delta V(X)| \leq c \sup _{U}|X|$ for any $X \in \mathrm{C}_{\mathrm{c}}^{1}\left(U, \mathbb{R}^{n}\right)$ with $\operatorname{spt}(X) \subset W$. By the Riesz representation theorem, there exist a Radon measure $\|\delta V\|$ on $U$ - the total variation measure of $\delta V$ - and a $\|\delta V\|$-measurable function $v: U \rightarrow \mathbb{R}^{n}$ with $|v|=1\|\delta V\|$-a.e. in $U$ satisfying

$$
\delta V(X)=-\int_{U}\langle\nu, X\rangle d\|\delta V\| \quad \forall X \in \mathrm{C}_{\mathrm{c}}^{1}\left(U, \mathbb{R}^{n}\right) .
$$

According to the Radon-Nikodym theorem, the limit

$$
D_{\|V\|}\|\delta V\|(x):=\lim _{r \rightarrow 0} \frac{\|\delta V\|\left(B_{r}(x)\right)}{\|V\|\left(B_{r}(x)\right)}
$$

exists for $\|V\|$-a.e. $x \in \mathbb{R}^{n}$. The mean curvature of $V$ is defined for $\|V\|$-almost every $x \in U$ as the vector

$$
H_{V}(x)=D_{\|V\|}\|\delta V\|(x) v(x) \equiv\left|H_{V}(x)\right| v(x) .
$$

It follows that, for every $X \in \mathrm{C}_{\mathrm{c}}^{1}\left(U, \mathbb{R}^{n}\right)$,

$$
\delta V(X)=-\int_{U}\left\langle H_{V}, X\right\rangle d\|V\|-\int_{U}\langle\nu, X\rangle d\|\delta V\|_{s},
$$

where $\|\delta V\|_{s}:=\|\delta V\|\left\llcorner\mathscr{B}_{V}\right.$, with $\mathscr{B}_{V}:=\left\{x \in U: D_{\|V\|}\|\delta V\|(x)=+\infty\right\}$. 
A varifold $V$ is said to have mean curvature in $\mathrm{L}^{p}$ if $H_{V} \in \mathrm{L}^{p}(\|V\|)$ and $\|\delta V\|$ is absolutely continuous with respect to $\|V\|$. In other words,

$$
H_{V} \in \mathrm{L}^{p} \Leftrightarrow\left\{\begin{array}{l}
H_{V} \in \mathrm{L}^{p}(\|V\|) \\
\delta V(X)=-\int_{U}\left\langle H_{V}, X\right\rangle d\|V\| \quad \text { for every } X \in \mathrm{C}_{\mathrm{c}}^{1}\left(U, \mathbb{R}^{n}\right) .
\end{array}\right.
$$

When $M$ is a smooth $k$-dimensional submanifold of $\mathbb{R}^{n}$, with $(\bar{M} \backslash M) \cap U=\emptyset$, the divergence theorem on manifolds implies that the mean curvature of the varifold $\mathbf{v}\left(M, \theta_{0}\right)$ for any positive constant $\theta_{0}$ is exactly the classical mean curvature of $M$, which can be calculated as

$$
H(x)=-\sum_{j} \operatorname{div}_{M} v_{j}(x) v_{j}(x)
$$

where $\left\{v_{j}(x)\right\}_{j}$ is an orthonormal frame for the orthogonal space $\left(T_{x} M\right)^{\perp}$.

We recall the coarea formula (see $[19,10]$ ) for rectifiable sets in $\mathbb{R}^{n}$ and mappings from $\mathbb{R}^{n}$ to $\mathbb{R}^{m}, m<n$. Let $M$ be a $k$-rectifiable set in $\mathbb{R}^{n}$ with $k \geq m, \theta: M \rightarrow$ $[0,+\infty]$ a Borel function, and $f: U \rightarrow \mathbb{R}^{m}$ a Lipschitz mapping defined on an open set $U \subset \mathbb{R}^{n}$. Then,

$$
\int_{x \in M \cap U} J_{M} f(x) \theta(x) d \mathscr{H}^{k}(x)=\int_{\mathbb{R}^{m}} \int_{y \in f^{-1}(t) \cap M} \theta(y) d \mathscr{H}^{k-m}(y) d \mathscr{H}^{m}(t),
$$

where $J_{M} f(x)$ denotes the tangential coarea factor of $f$ at $x \in M$, defined for $\mathscr{H}^{k}$ almost every $x \in M$ by

$$
J_{M} f(x)=\sqrt{\operatorname{det}\left(\nabla^{M} f(x) \cdot \nabla^{M} f(x)^{t}\right)} .
$$

We also recall Allard's isoperimetric inequality for varifolds (see [1]).

Theorem 1.1 (Isoperimetric inequality for varifolds). There exists a constant $C>0$ such that, for every $k$-varifold $V$ with locally bounded first variation and for every smooth function $\varphi \geq 0$ with compact support in $\mathbb{R}^{n}$,

$\int_{E_{\varphi}} \varphi d\|V\| \leq C\left(\int_{\mathbb{R}^{n}} \varphi d\|V\|\right)^{\frac{1}{k}}\left(\int_{\mathbb{R}^{n}} \varphi d\|\delta V\|+\int_{\mathbb{R}^{n} \times \mathrm{G}_{n, k}}\left|\nabla^{S} \varphi\right| d V\right)$,

where $E_{\varphi}=\left\{x: \varphi(x) \Theta^{k}(\|V\|, x) \geq 1\right\}$.

\section{Integral 1-varifolds with locally bounded first variation}

\subsection{Locality property of the generalized curvature}

We consider integral 1-varifolds of type $V=\mathbf{v}(M, \theta)$ in $U \subset \mathbb{R}^{n}$, where $M \subset U$ is a 1 -rectifiable set and $\theta \geq 1$ is an integer-valued Borel function on $M$. Thus, 
$\|V\|=\theta \mathscr{H}^{1}\llcorner M$ is a Radon measure on $U$ and we assume in addition that $V$ has a locally bounded first variation, that is, for any smooth vectorfield $X \in C_{c}^{1}\left(\mathbb{R}^{n} ; \mathbb{R}^{n}\right)$

$$
\delta V(X)=\int_{M} \operatorname{div}_{M} X d\|V\|=-\int_{M}\langle\kappa, X\rangle d\|V\|+\delta V_{S}(X),
$$

where $\delta V_{s}$ denotes the singular part of the first variation with respect to the weight measure $\|V\|$. We now prove the following

Theorem 2.1. Let $V_{1}=\mathbf{v}\left(M_{1}, \theta_{1}\right), V_{2}=\mathbf{v}\left(M_{2}, \theta_{2}\right)$ be two integral 1-varifolds with locally bounded first variation. Then, denoting by $\kappa_{1}, \kappa_{2}$ their respective curvatures, one has $\kappa_{1}(x)=\kappa_{2}(x)$ for $\mathscr{H}^{1}$-almost every $x \in S=M_{1} \cap M_{2}$.

Proof. Let $x \in S$ satisfy the following properties:

(i) $x$ is a point of density 1 for $M_{1}, M_{2}$ and $S$;

(ii) $x$ is a Lebesgue point for $\theta_{i}$ and $\kappa_{i} \theta_{i}(i=1,2)$;

(iii) $\lim _{r \rightarrow 0} \frac{\left\|\delta V_{s}\right\|\left(B_{r}(x)\right)}{\|V\|\left(B_{r}(x)\right)}=0$ for $V=V_{1}, V_{2}$.

In particular, this means

$$
\begin{aligned}
& \lim _{r \rightarrow 0} \frac{\mathscr{H}^{1}\left(\left(M_{i} \backslash S\right) \cap B_{r}(x)\right)}{2 r}=0, \\
& \lim _{r \rightarrow 0} \frac{1}{2 r} \int_{y \in M_{i} \cap B_{r}(x)}\left|\theta_{i}(y)-\theta_{i}(x)\right| d \mathscr{H}^{1}(y)=0, \\
& \lim _{r \rightarrow 0} \frac{1}{2 r} \int_{y \in M_{i} \cap B_{r}(x)}\left|\kappa_{i}(y) \theta_{i}(y)-\kappa_{i}(x) \theta_{i}(x)\right| d \mathscr{H}^{1}(y)=0
\end{aligned}
$$

for $i=1,2$ and with $B_{r}(x)$ denoting the ball of radius $r$ and center $x$. Recall that $\mathscr{H}^{1}$-a.e. $x \in S$ has such properties. Without loss of generality, we may assume that $x=0$ and we shall denote in the sequel $B_{r}=B_{r}(0)$. In view of Property (iii) above, we may also neglect the singular part, i.e. assume that the varifolds have curvatures in $\mathrm{L}_{\text {loc }}^{1}$.

Let us write the coarea formula (1.5) with $f(x)=|x|, M=M_{i} \backslash S$ and $\theta=\theta_{i}$, also observing that

$$
J_{M} f(x)=\left|\nabla^{M} f(x)\right|=\frac{\left|x_{M}\right|}{|x|} \leq 1
$$

where $x_{M}$ denotes the projection of $x$ onto the tangent line $T_{x} M$. We obtain the inequality

$$
\left\|V_{i}\right\|\left(\left(M_{i} \backslash S\right) \cap B_{r}\right) \geq \int_{0}^{r} \int_{\left(M_{i} \backslash S\right) \cap \partial B_{t}} \theta_{i} d \mathscr{H}^{0} d t, \quad i=1,2 .
$$


By combining (2.1) and (2.2), one can show that

$$
\frac{\left\|V_{i}\right\|\left(\left(M_{i} \backslash S\right) \cap B_{r}\right)}{2 r}=\frac{1}{2 r} \int_{\left(M_{i} \backslash S\right) \cap B_{r}} \theta_{i} d \mathscr{H}^{1} \longrightarrow 0 \quad \text { as } r \rightarrow 0, \quad i=1,2,
$$

hence if we define

$$
g_{i}(t)=\int_{\left(M_{i} \backslash S\right) \cap \partial B_{t}} \theta_{i} d \mathscr{H}^{0}, \quad i=1,2,
$$

we find by (2.4) and (2.5) that 0 is a point of density 1 for the set $\mathcal{T}_{i}=\{t>0$ : $\left.g_{i}(t)=0\right\}$, that is,

$$
\lim _{r \rightarrow 0} \frac{\left|\mathcal{T}_{i} \cap[0, r)\right|}{r}=1 .
$$

Therefore, by the fact that the measure $\mathscr{H}^{0}$ is integer-valued we can find a decreasing sequence $\left(r_{k}\right)_{k}$ converging to 0 and such that $r_{k}$ is a Lebesgue point for both $g_{1}$ and $g_{2}$, with $g_{1}\left(r_{k}\right)=g_{2}\left(r_{k}\right)=0$, thus

$$
\lim _{\epsilon \rightarrow 0} \frac{1}{\epsilon} \int_{r_{k}-\epsilon}^{r_{k}} g_{i}(t) d t=0 \quad \forall i=1,2 .
$$

By arguing exactly in the same way, we can also assume that

$$
\lim _{\epsilon \rightarrow 0} \frac{1}{\epsilon} \int_{r_{k}-\epsilon}^{r_{k}} h(t) d t=0
$$

where

$$
h(t)=\int_{y \in S \cap \partial B_{t}}\left|\theta_{1}(0) \theta_{2}(y)-\theta_{2}(0) \theta_{1}(y)\right| d \mathscr{H}^{0}(y) .
$$

Indeed, one can observe as before that the set

$$
\mathcal{Q}=\{t>0: h(t)=0\}
$$

has density 1 at $t=0$, as it follows from the integrality of the multiplicity functions combined with coarea formula and

$$
\lim _{r \rightarrow 0} \frac{1}{2 r} \int_{S \cap B_{r}}\left|\theta_{1}(0) \theta_{2}-\theta_{2}(0) \theta_{1}\right| d \mathscr{H}^{1}=0,
$$

this last equality being a consequence of (2.2). Therefore, $r_{k}$ can be chosen in such a way that (2.7) holds, too. Now, for a given $\xi \in \mathbb{R}^{n}$ and $0<\epsilon<r_{k}$, we define the vector field $X_{k, \epsilon}(x)=\eta_{r_{k}, \epsilon}(|x|) \xi$, where $\eta_{r, \epsilon}$ is a $C^{1}$ function defined on $[0,+\infty)$, with support contained in $[0, r)$ and such that

$$
\eta_{r, \epsilon}(t)=1 \quad \text { if } 0 \leq t \leq r-\epsilon, \quad\left\|\eta_{r, \epsilon}^{\prime}\right\|_{\infty} \leq \frac{2}{\epsilon} .
$$


By applying the coarea formula (1.5) and recalling that $\nabla^{M_{i}}|x|=x_{M_{i}} /|x|$, we get

$$
\begin{aligned}
\int_{M_{i} \backslash S}\left|\eta_{r_{k}, \epsilon}^{\prime} \nabla^{M_{i}}\right| x|| \theta_{i} d \mathscr{H}^{1} & \leq \frac{2}{\epsilon} \int_{r_{k}-\epsilon}^{r_{k}} \int_{\partial B_{t} \cap\left(M_{i} \backslash S\right)} \theta_{i} d \mathscr{H}^{0} d t \\
& =\frac{2}{\epsilon} \int_{r_{k}-\epsilon}^{r_{k}} g_{i}(t) d t .
\end{aligned}
$$

Combining this last inequality with (2.6) and

$$
\operatorname{div}_{M_{i}} X_{k, \epsilon}(x)=\eta_{r_{k}, \epsilon}^{\prime}(x)\left\langle\xi, \frac{x_{M_{i}}}{|x|}\right\rangle
$$

implies

$$
\lim _{\epsilon \rightarrow 0} \int_{\left(M_{i} \backslash S\right) \cap B_{r_{k}}} \operatorname{div}_{M_{i}} X_{k, \epsilon} d\left\|V_{i}\right\|=0, \quad \forall i=1,2, \quad \forall k .
$$

At this point, we only need to show that the scalar product $\Delta=\left\langle\kappa_{1}(0)-\kappa_{2}(0), \xi\right\rangle$ cannot be positive, thus it has to be zero by the arbitrary choice of $\xi$. First, thanks to (2.3) we get

$$
\Delta=\frac{1}{\theta_{1}(0) \theta_{2}(0)} \lim _{k}\left(\frac{\theta_{2}(0)}{2 r_{k}} \int_{M_{1} \cap B_{r_{k}}}\left\langle\xi, \kappa_{1}\right\rangle d\left\|V_{1}\right\|-\frac{\theta_{1}(0)}{2 r_{k}} \int_{M_{2} \cap B_{r_{k}}}\left\langle\xi, \kappa_{2}\right\rangle d\left\|V_{2}\right\|\right),
$$

and, owing to the Dominated Convergence Theorem,

$$
\begin{aligned}
\Delta=\frac{1}{\theta_{1}(0) \theta_{2}(0)} \lim _{k} \lim _{\epsilon \rightarrow 0}\left(\frac{\theta_{2}(0)}{2 r_{k}} \int_{M_{1} \cap B_{r_{k}}}\left\langle X_{k, \epsilon}, \kappa_{1}\right\rangle d\left\|V_{1}\right\|\right. & \\
& \left.-\frac{\theta_{1}(0)}{2 r_{k}} \int_{M_{2} \cap B_{r_{k}}}\left\langle X_{k, \epsilon}, \kappa_{2}\right\rangle d\left\|V_{2}\right\|\right) .
\end{aligned}
$$

Therefore, by the definition of the generalized curvature we immediately infer that

$$
\begin{aligned}
\Delta=\frac{1}{\theta_{1}(0) \theta_{2}(0)} \lim _{k} \lim _{\epsilon \rightarrow 0}\left(-\frac{\theta_{2}(0)}{2 r_{k}}\right. & \int_{M_{1} \cap B_{r_{k}}} \operatorname{div}_{M_{1}} X_{k, \epsilon} d\left\|V_{1}\right\| \\
& \left.+\frac{\theta_{1}(0)}{2 r_{k}} \int_{M_{2} \cap B_{r_{k}}} \operatorname{div}_{M_{2}} X_{k, \epsilon} d\left\|V_{2}\right\|\right) .
\end{aligned}
$$

Noticing that $\operatorname{div}_{S} G(x)=\operatorname{div}_{M_{1}} G(x)=\operatorname{div}_{M_{2}} G(x)$ for $\mathscr{H}^{1}$-almost all $x \in S$, and thanks to (2.8), one can rewrite (2.9) as

$$
\Delta=\frac{1}{\theta_{1}(0) \theta_{2}(0)} \lim _{k} \lim _{\epsilon \rightarrow 0}\left(\frac{1}{2 r_{k}} \int_{S \cap B_{r_{k}}} \operatorname{div}_{S} X_{k, \epsilon}\left(\theta_{1}(0) \theta_{2}-\theta_{2}(0) \theta_{1}\right) d \mathscr{H}^{1}\right) .
$$


Computing the tangential divergence of $X_{k, \epsilon}$ and, then, using the coarea formula (1.5) in (2.10), gives

$$
\Delta \leq \frac{|\xi|}{\theta_{1}(0) \theta_{2}(0)} \lim _{k} \frac{1}{r_{k}} \lim _{\epsilon \rightarrow 0} \frac{1}{\epsilon} \int_{r_{k}-\epsilon}^{r_{k}} h(t) d t=0 .
$$

We conclude that $\Delta=0$, hence $\kappa_{1}(0)=\kappa_{2}(0)$, as wanted.

A straightforward consequence of Theorem 2.1 is the following

Corollary 2.2. Let $V=\mathbf{v}(M, \theta)$ be an integral 1-varifold in $U \subset \mathbb{R}^{n}$, with locally bounded first variation. Then the vector $\kappa(x)$ coincides with the classical curvature of any $C^{2}$ curve $\gamma$, for $\mathscr{H}^{1}$-almost all $x \in \gamma \cap \mathrm{spt}\|V\|$.

\subsection{A 1-varifold with curvature in $L^{1} \backslash L^{p}$ for all $p>1$}

Here we construct an integral 1 -varifold in $\mathbb{R}^{2}$ with curvature in $\mathrm{L}^{1} \backslash \mathrm{L}^{p}$ for any $p>1$. This varifold is obtained as the limit of a sequence of graphs of smooth functions, its support is $C^{2}$-rectifiable (i.e., covered up to a negligible set by a countable union of $\mathrm{C}^{2}$ curves, see $[5,18]$ ) and, due to our Theorem 2.1 , its curvature coincides $\mathscr{H}^{1}$-almost everywhere with the classical one, as stated in Corollary 2.2 above.

Let $\zeta \in C^{2}([0,1])$ with $\zeta \neq 0$ and

$$
\zeta(0)=\zeta^{\prime}(0)=\zeta(1)=\zeta^{\prime}(1)=0 .
$$

Given $\lambda>0$ and $0 \leq a<b \leq 1$, define

$$
\zeta_{a, b, \lambda}(t)= \begin{cases}\lambda \zeta\left(\frac{t-a}{b-a}\right) & \text { if } t \in[a, b] \\ 0 & \text { otherwise. }\end{cases}
$$

Let $\left(a_{n}, b_{n}\right)_{n \geq 2}$ be a sequence of nonempty, open and mutually disjoint subintervals of $[0,1]$, such that $b_{n}-a_{n} \leq 2^{-n}$ and

$$
0<\sum_{n \geq 2}\left(b_{n}-a_{n}\right)<1 .
$$

In particular, the set $C=[0,1] \backslash \bigcup_{n}\left(a_{n}, b_{n}\right)$ is closed and has positive $\mathscr{L}^{1}$ measure. We denote by $\left(\lambda_{n}\right)_{n}$ a sequence of positive real numbers, that will be chosen later, and we set

$$
\zeta_{n}(t)=\zeta_{a_{n}, b_{n}, \lambda_{n}}(t)
$$

for $t \in[0,1]$ and $n \geq 2$. Then, we compute the integral of the $p$-th power of the curvature of the graph of $\zeta_{n}$ over the graph itself, that is,

$$
K_{n}^{p}=\int_{a_{n}}^{b_{n}} \frac{\left|\zeta_{n}^{\prime \prime}(t)\right|^{p}}{\left[1+\zeta_{n}^{\prime}(t)^{2}\right]^{\frac{3 p-1}{2}}} d t
$$


Since

$$
\begin{aligned}
& \zeta_{n}^{\prime}(t)=\frac{\lambda_{n}}{b_{n}-a_{n}} \zeta^{\prime}\left(\frac{t-a_{n}}{b_{n}-a_{n}}\right), \\
& \zeta_{n}^{\prime \prime}(t)=\frac{\lambda_{n}}{\left(b_{n}-a_{n}\right)^{2}} \zeta^{\prime \prime}\left(\frac{t-a_{n}}{b_{n}-a_{n}}\right),
\end{aligned}
$$

and choosing $0 \leq \lambda_{n} \leq b_{n}-a_{n}$, we infer that the Lipschitz constant of $\zeta_{n}$ is bounded by that of $\zeta$, for all $n \geq 2$. Therefore, there exists a uniform constant $c \geq 1$ such that

$$
c^{-1} K_{n}^{p} \leq \int_{a_{n}}^{b_{n}}\left|\zeta_{n}^{\prime \prime}(t)\right|^{p} d t \leq c K_{n}^{p}
$$

and therefore

$$
c^{-1} K_{n}^{p} \leq K \frac{\lambda_{n}^{p}}{\left(b_{n}-a_{n}\right)^{2 p-1}} \leq c K_{n}^{p},
$$

where

$$
K=\int_{0}^{1}\left|\zeta^{\prime \prime}(t)\right|^{p} d t>0
$$

At this point, we look for $\lambda_{n}$ satisfying

(i) $0<\lambda_{n} \leq b_{n}-a_{n}$,

(ii) $\sum_{n \geq 2} K_{n}^{p}<+\infty$ if and only if $p=1$.

A possible choice for $\lambda_{n}$ is given by

$$
\lambda_{n}=\frac{b_{n}-a_{n}}{n^{2}} .
$$

Indeed, up to multiplicative constants one gets

$$
\sum_{n} K_{n}^{1}=\sum_{n} \frac{1}{n^{2}}<+\infty
$$

and

$$
\sum_{n} K_{n}^{p} \geq \sum_{n} \frac{2^{n(p-1)}}{n^{2 p}}=+\infty
$$

for all $p>1$. Now, define for $t \in \mathbb{R}$

$$
\eta(t)=\sum_{n} \zeta_{n}(t)
$$

Thanks to (2.11) and (2.12), the 1-varifold $V=\mathbf{v}(G, 1)$ associated to the graph $G$ of $\eta$ has curvature in $L^{1} \backslash L^{p}$ for all $p>1$. Indeed, setting $\eta_{N}=\sum_{n=2}^{N} \zeta_{n}$ and letting 
$G_{N}$ be the graph of $\eta_{N}$, one can verify that the 1-rectifiable varifolds $V_{N}=\mathbf{v}\left(G_{N}, 1\right)$ weakly converge to $V$ as $N \rightarrow \infty$, and the same happens for the respective first variations:

$$
\delta V_{N} \underset{N}{\rightarrow} \delta V
$$

thus for any open set $A \subset(0,1) \times \mathbb{R}$ one has

$$
\|\delta V\|(A) \leq \lim _{N}\left\|\delta V_{N}\right\|(A)=\lim _{N} \int_{A}\left|\kappa_{N}\right| \mathbb{1}_{G_{N}} d\|V\|=\int_{A}|\kappa| d\|V\|,
$$

where $\mathbb{1}_{G_{N}}$ is the characteristic function of $G_{N}$ and for $(x, y) \in G$ we define $\kappa(x, y)=$ $\kappa_{N}(x, y)$ for $N$ large enough and $y>0$ (the definition is well posed, since the intervals $\left(a_{n}, b_{n}\right)$ are pairwise disjoint) and $\kappa(x, y)=0$ whenever $y=0$. This shows that $V$ has curvature in $\mathrm{L}^{1}$. It is also evident from (2.12) that the curvature of $V$ cannot belong to $\mathrm{L}^{p}$ for $p>1$. Lastly, the $\mathrm{C}^{2}$-rectifiability comes from $\mathscr{H}^{1}\left(\operatorname{spt} V \backslash \bigcup_{N \geq 2} G_{N}\right)=0$.

An example of the construction of such varifold $V$ is illustrated in Figure 1.
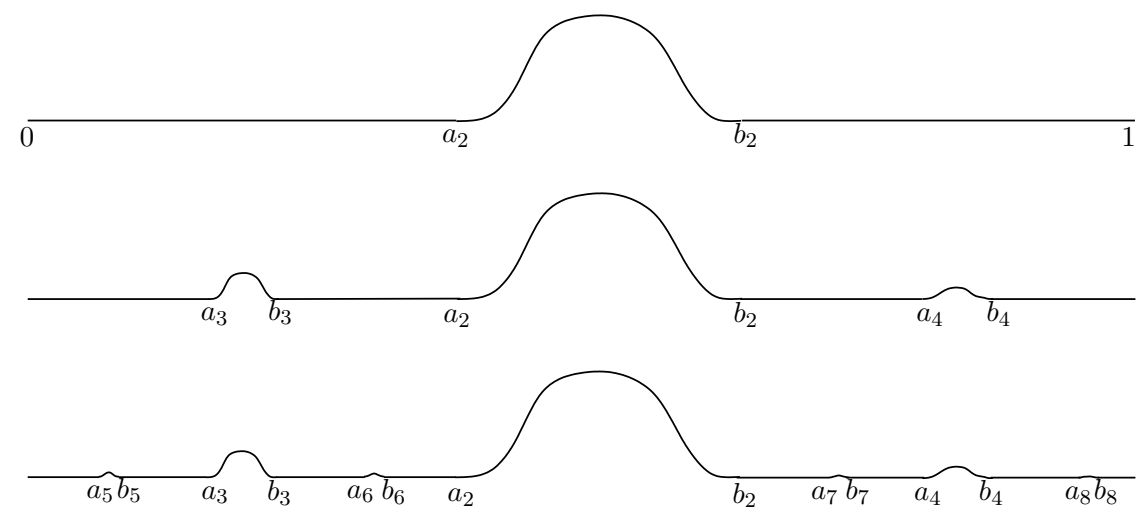

Figure 1. As a particular example, we take the sequence $\left(a_{n}, b_{n}\right)$ of all middle intervals in $[0,1]$ of size $2^{-2 p-2}$ whenever $2^{p}<n \leq 2^{p+1}, p=0,1,2, \ldots$. The union of these intervals is the complement of a Cantor-type set $C$ with positive measure $\mathscr{H}^{1}(C)=\frac{1}{2}$. We have represented from top to bottom the functions $\zeta_{2}, \sum_{n=2}^{4} \zeta_{n}$ and $\sum_{n=2}^{8} \zeta_{n}$.

\section{Rectifiable $k$-varifolds with locally bounded first variation}

\subsection{Relative isoperimetric inequalities for $\boldsymbol{k}$-varifolds}

The isoperimetric inequality for varifolds due to W. K. Allard [1] is recalled in Theorem 1.1. We derive from it the following differential inequalities, that will be useful for studying the locality of rectifiable $k$-varifolds. 
Proposition 3.1 (Relative isoperimetric inequalities). Let $V$ be a $k$-varifold in $\mathbb{R}^{n}$, and let $A \subset \mathbb{R}^{n}$ be an open, bounded set with Lipschitz boundary. Then,

$$
\|V\|(A)^{\frac{k-1}{k}} \leq C\left(\|\delta V\|(A)-\left.D_{+}\|V\|\left(A \backslash A_{\epsilon}\right)\right|_{\epsilon=0}\right),
$$

where $A_{\epsilon}$ is the set of points of $A$ whose distance from $\mathbb{R}^{n} \backslash A$ is less than $\epsilon$, and $\left.D_{+}\|V\|\left(A \backslash A_{\epsilon}\right)\right|_{\epsilon=0}$ denotes the lower right derivative of the non-increasing function $\epsilon \rightarrow\|V\|\left(A \backslash A_{\epsilon}\right)$ at $\epsilon=0$.

Moreover, if we define $g(r)=\|V\|\left(B_{r}\right)$, then $g$ is a non-decreasing (thus almost everywhere differentiable) function, and it holds

$$
g(r)^{\frac{k-1}{k}} \leq C\left(\|\delta V\|\left(B_{r}\right)+g^{\prime}(r)\right) \quad \text { for almost all } r>0 .
$$

Proof. Let $\epsilon>0$ and let $\varphi_{\epsilon}: A \rightarrow \mathbb{R}$ be defined as

$$
\varphi_{\epsilon}(x)=\min \left(\epsilon^{-1} d\left(x, \mathbb{R}^{n} \backslash A\right), 1\right) .
$$

Clearly, $\varphi_{\epsilon}$ is a Lipschitz function with compact support in $\mathbb{R}^{n}$. Approximating $\varphi_{\epsilon}$ by a sequence of non-negative, $\mathrm{C}^{1}$ functions with compact support in $\mathbb{R}^{n}$, it follows from Allard's isoperimetric inequality (1.6) that

$$
\int_{E_{\varphi_{\epsilon}}} \varphi_{\epsilon} d\|V\| \leq C\left(\int_{\mathbb{R}^{n}} \varphi_{\epsilon} d\|V\|\right)^{\frac{1}{k}}\left(\int_{\mathbb{R}^{n}} \varphi_{\epsilon} d\|\delta V\|+\int_{\mathbb{R}^{n} \times G(n, k)}\left|\nabla^{S} \varphi_{\epsilon}\right| d V\right),
$$

where $E_{\varphi_{\epsilon}}=\left\{x: \varphi_{\epsilon}(x) \Theta^{k}(\|V\|, x) \geq 1\right\}$. Moreover, we have

$$
\left|\nabla \varphi_{\epsilon}(x)\right| \leq \frac{1}{\epsilon} \quad \text { on } A_{\epsilon}:=\left\{x \in A: d\left(x, \mathbb{R}^{n} \backslash A\right) \leq \epsilon\right\},
$$

and therefore (3.3) can be rewritten as

$$
\int_{A \backslash A_{\epsilon}} d\|V\| \leq \int_{E_{\varphi_{\epsilon}}} \varphi_{\epsilon} d\|V\| \leq C\left(\int_{A} d\|V\|\right)^{\frac{1}{k}}\left(\int_{A} d\|\delta V\|+\frac{1}{\epsilon} \int_{A_{\epsilon}} d\|V\|\right) .
$$

Now, since

$$
\lim _{\epsilon \rightarrow 0} \int_{A \backslash A_{\epsilon}} d\|V\|=\int_{A} d\|V\|
$$

and

$$
\frac{1}{\epsilon} \int_{A_{\epsilon}} d\|V\|=\frac{\|V\|\left(A_{\epsilon}\right)}{\epsilon}=-\frac{\|V\|\left(A \backslash A_{\epsilon}\right)-\|V\|(A)}{\epsilon},
$$

the Dominated Convergence Theorem allows us to take the limit in (3.4) as $\epsilon \rightarrow 0$, yielding

$$
\|V\|(A)^{\frac{k-1}{k}} \leq C\left(\|\delta V\|(A)-\left.D_{+}\|V\|\left(A \backslash A_{\epsilon}\right)\right|_{\epsilon=0}\right) .
$$


Take now $A \equiv B_{r}$ and remark that $A \backslash A_{\epsilon}=B_{r-\epsilon}$. Denoting $g(r)=\|V\|\left(B_{r}\right)$, we deduce from the monotonicity of $g$ that it is almost everywhere differentiable. In particular, for almost every $r>0$, and using the fact that $g(r-\epsilon)-g(r)=-\|V\|\left(A_{\epsilon}\right)$ for almost every $r>0$ (and for every $\epsilon>0$ ), we get

$$
g^{\prime}(r)=-\lim _{\epsilon \rightarrow 0} \frac{g(r-\epsilon)-g(r)}{\epsilon}=\lim _{\epsilon \rightarrow 0} \frac{\|V\|\left(A_{\epsilon}\right)}{\epsilon} .
$$

Then, (3.2) immediately follows from (3.5) and (3.1).

\subsection{A locality result for rectifiable $k$-varifolds}

First, we derive a useful formula for computing the mean curvature of a rectifiable $k$-varifold. This formula will be crucial in the proof of our second locality result (Theorem 3.4). More precisely, given a rectifiable $k$-varifold $V=\mathbf{v}(M, \theta)$ with locally bounded first variation, we show in the next proposition that the integral of the mean curvature on a ball $B_{r}$ essentially coincides with the integral on the sphere $\partial B_{r}$ of the conormal $\eta$ to $M$, up to an error term due to the singular part of the first variation. Therefore, we obtain an equivalent expression for the curvature at a Lebesgue point $x_{0} \in M$. Recall that $x_{M}$ denotes the orthogonal projection of $x$ onto $T_{x} M$.

Proposition 3.2. Let $x_{0} \in \mathbb{R}^{n}$ and $V=\mathbf{v}(M, \theta)$ be a rectifiable $k$-varifold with locally bounded first variation. Then, setting $\sigma=\theta \mathscr{H}^{k-1}\llcorner M$, we get for almost every $r>0$

$$
\left|\int_{B_{r}\left(x_{0}\right)} H d\|V\|+\int_{\partial B_{r}\left(x_{0}\right)} \eta d \sigma\right| \leq\|\delta V\|_{s}\left(B_{r}\left(x_{0}\right)\right),
$$

where

$$
\eta(x)= \begin{cases}-\frac{x_{M}}{\left|x_{M}\right|} & \text { if }\left|x_{M}\right| \neq 0 \\ 0 & \text { elsewhere }\end{cases}
$$

is the inner conormal to $M \cap B_{r}\left(x_{0}\right)$ at $x \in M \cap \partial B_{r}\left(x_{0}\right)$. Consequently, if $x_{0} \in M$ is a Lebesgue point for $H$, then

$$
H\left(x_{0}\right)=-\lim _{r \rightarrow 0^{+}} \frac{1}{\|V\|\left(B_{r}\left(x_{0}\right)\right)} \int_{\partial B_{r}\left(x_{0}\right)} \eta d \sigma .
$$

Proof. For simplicity, we assume that $x_{0}=0$. Let us consider a Lipschitz cutoff function $\beta_{\epsilon}:[0,+\infty) \rightarrow \mathbb{R}$ such that $\beta_{\epsilon}(t)=1$ for $t \in[0, r-\epsilon], \beta_{\epsilon}(t)=1-\frac{t-r+\epsilon}{\epsilon}$ for $t \in(r-\epsilon, r]$ and $\beta_{\epsilon}(t)=0$ elsewhere. Then, choose a unit vector $w \in \mathbb{R}^{n}$ and define the vector field $X_{\epsilon}=\beta_{\epsilon}(|x|) w$. The definition of the generalized mean curvature yields

$$
\int_{B_{r}} \operatorname{div}_{M} X_{\epsilon} d\|V\|=-\int_{B_{r}} \beta_{\epsilon}\langle H, w\rangle d\|V\|+\delta V_{s}\left(X_{\epsilon}\right),
$$


and, thanks to our assumptions, we also have

$$
\int_{B_{r}} \operatorname{div}_{M} X_{\epsilon} d\|V\|=-\frac{1}{\epsilon} \int_{B_{r} \backslash B_{r-\epsilon}} \frac{\left\langle x_{M}, w\right\rangle}{|x|} d\|V\| .
$$

By the Dominated Convergence Theorem,

$$
\lim _{\epsilon \rightarrow 0} \int_{B_{r}}\langle H, w\rangle \beta_{\epsilon} d\|V\|=\int_{B_{r}}\langle H, w\rangle d\|V\|, \quad \forall r>0 .
$$

Therefore, the derivative

$$
\frac{d}{d r} \int_{B_{r}} \frac{\left\langle x_{M}, w\right\rangle}{|x|} d\|V\|
$$

exists for almost all $r>0$ as the limit of the difference quotient

$$
\frac{1}{\epsilon} \int_{B_{r} \backslash B_{r-\epsilon}} \frac{\left\langle x_{M}, w\right\rangle}{|x|} d\|V\|
$$

and, in view of (1.3), one has

$$
\frac{d}{d r} \int_{B_{r}} \frac{\left\langle x_{M}, w\right\rangle}{|x|} d\|V\|=\int_{B_{r}}\langle H, w\rangle d\|V\|+\int_{B_{r}}\langle v, w\rangle d\left\|\delta V_{s}\right\| .
$$

Observe now that, denoting $N:=\left\{x:\left|x_{M}\right|=0\right\}$, the coarea formula (1.5) gives

$$
\begin{aligned}
\int_{B_{r}} \frac{\left\langle x_{M}, w\right\rangle}{|x|} d\|V\| & =\int_{B_{r} \backslash N} \frac{\left\langle x_{M}, w\right\rangle}{\left|x_{M}\right|} \frac{\left|x_{M}\right|}{|x|} d\|V\| \\
& =\int_{0}^{r} \int_{\partial B_{t} \backslash N} \frac{\left\langle x_{M}, w\right\rangle}{\left|x_{M}\right|} d \sigma d t .
\end{aligned}
$$

We deduce that, for every Lebesgue point of the integrable function

$$
t \mapsto \int_{\partial B_{t} \backslash N} \frac{\left\langle x_{M}, w\right\rangle}{\left|x_{M}\right|} d \sigma,
$$

one gets

$$
\frac{d}{d r} \int_{B_{r}} \frac{\left\langle x_{M}, w\right\rangle}{|x|} d\|V\|=\int_{\partial B_{r} \backslash N} \frac{\left\langle x_{M}, w\right\rangle}{\left|x_{M}\right|} d \sigma .
$$

By the definition of the conormal $\eta$, we conclude that, for every vector $w \in \mathbb{R}^{n}$,

$$
\left|\int_{B_{r}}\langle H, w\rangle d\|V\|+\int_{\partial B_{r}}\langle\eta, w\rangle d \sigma\right| \leq|w|\left\|\delta V_{s}\right\|\left(B_{r}\right), \quad \text { for a.e. } r>0,
$$

or, equivalently,

$$
\left|\int_{B_{r}} H d\|V\|+\int_{\partial B_{r}} \eta d \sigma\right| \leq\left\|\delta V_{s}\right\|\left(B_{r}\right), \quad \text { for a.e. } r>0 .
$$


This proves (3.6) and, since

$$
\frac{\left\|\delta V_{s}\right\|\left(B_{r}\right)}{\|V\|\left(B_{r}\right)} \rightarrow 0 \quad \text { as } r \rightarrow 0
$$

also (3.7) follows.

Remark 3.3. In case $\delta V$ has no singular part with respect to $\|V\|$, (3.6) becomes

$$
\int_{B_{r}\left(x_{0}\right)} H d\|V\|=-\int_{\partial B_{r}\left(x_{0}\right)} \eta d \sigma, \quad \text { for almost every } r>0 .
$$

Below we prove a locality property for $k$-varifolds in $\mathbb{R}^{n}, k \geq 2$, requiring some extra hypotheses on the varifolds under consideration. The proof is quite different from that of Theorem 2.1, mainly because the Hausdorff measure $\mathscr{H}^{k-1}$ is no more a discrete (counting) measure. Our result gives a positive answer to the locality problem in any dimension $k \geq 2$ and any codimension, assuming that the support of one of the two varifolds is locally contained into the other, and also that the two multiplicities are locally constant on the intersection of the supports.

Theorem 3.4. Let $V_{i}=\mathbf{v}\left(M_{i}, \theta_{i}\right), i=1,2$, be two rectifiable $k$-varifolds in $U \subset \mathbb{R}^{n}$ with locally bounded first variations, and let $H_{1}, H_{2}$ denote their respective mean curvatures. Suppose that there exists an open set $A \subset U$ such that

(i) $M_{1} \cap A \subset M_{2}$,

(ii) $\theta_{1}(x)$ and $\theta_{2}(x)$ are $\mathscr{H}^{k}$-a.e. constant on $M_{1} \cap A$.

Then, $H_{1}(x)=H_{2}(x)$ for $\mathscr{H}^{k}$-a.e. $x \in M_{1} \cap A$.

Proof. Up to multiplication by suitable constants, we may assume without loss of generality that $\theta_{1}(x)=\theta_{2}(x)=\theta_{0}$ constant, for $\mathscr{H}^{k}$-almost every $x \in M_{1} \cap A$. Moreover, the theory of rectifiable sets and of rectifiable measures ensures that $\mathscr{H}^{k}$ a.e. point $x \in M_{1} \cap A$ is generic in the sense that it satisfies

(i) $\Theta^{k}\left(\left\|V_{i}\right\|\left\llcorner\left(M_{2} \backslash M_{1}\right), x\right)=0\right.$ and $\Theta^{k}\left(\left\|V_{i}\right\|, x\right)=\theta_{0}$ for $i=1,2$;

(ii) $x$ is a Lebesgue point for $H_{1}$ and $H_{2}$;

(iii) $\left\|\delta V_{s}\right\|\left(B_{r}(x)\right)=\mathrm{o}\left(\|V\|\left(B_{r}(x)\right)\right)$ for $V=V_{1}, V_{2}$.

Suppose, without loss of generality, that $x=0$ is a generic point of $M_{1} \cap A$. Let $r_{0}$ be such that $B_{r_{0}}:=B_{r_{0}}(0) \subset A$, let $\widetilde{M}_{2}=M_{2} \backslash M_{1}$ and $\widetilde{V}_{2}=\mathbf{v}\left(\widetilde{M}_{2}, \theta_{2}\right)$. Obviously, $\widetilde{V}_{2}$ is a rectifiable $k$-varifold, but possibly $\delta \widetilde{V}_{2}$ has an extra singular part with respect to $\left\|\widetilde{V}_{2}\right\|$. By (3.6), for almost every $0<r<r_{0}$

$$
\begin{aligned}
\int_{B_{r}} H_{2} d\left\|V_{2}\right\|+\mathrm{o}\left(\left\|V_{2}\right\|\left(B_{r}\right)\right) & =-\int_{\partial B_{r}} \eta_{2} d \sigma_{2} \\
& =-\int_{\partial B_{r} \cap M_{1}} \eta_{2} d \sigma_{2}-\int_{\partial B_{r} \cap \widetilde{M}_{2}} \eta_{2} d \sigma_{2},
\end{aligned}
$$


where $\sigma_{2}=\theta_{2} \mathscr{H}^{k-1}\left\llcorner M_{2}\right.$. Since both $M_{1}$ and $M_{2}$ are rectifiable, they have the same tangent space at $\mathscr{H}^{k}$-almost every point of $M_{1} \cap A$, thus $\eta_{1}=\eta_{2}$ for $\mathscr{H}^{k}$-a.e. $x \in M_{1} \cap A$. Then, observe that the coarea formula and the assumption $\theta_{2}(x)=$ $\theta_{1}(x)=\theta_{0} \mathscr{H}^{k}$-almost everywhere on $M_{1} \cap A$ yield, for almost every $0<r<r_{0}$,

$$
\int_{\partial B_{r} \cap M_{1}}\left(\theta_{2}-\theta_{1}\right) d \mathscr{H}^{k-1}=0
$$

that is,

$$
\sigma_{2}\left(\partial B_{r} \cap M_{1}\right)=\sigma_{1}\left(\partial B_{r}\right)=\theta_{0} \mathscr{H}^{k-1}\left(\partial B_{r} \cap M_{1}\right),
$$

where $\sigma_{i}=\theta_{i} \mathscr{H}^{k-1}\left\llcorner M_{i}, i=1,2\right.$. We deduce by (3.6) that, for a.e. $0<r<r_{0}$,

$$
\int_{\partial B_{r} \cap M_{1}} \eta_{2} d \sigma_{2}=\int_{\partial B_{r}} \eta_{1} d \sigma_{1}=-\int_{B_{r}} H_{1} d\left\|V_{1}\right\|-\mathrm{o}\left(\left\|V_{1}\right\|\left(B_{r}\right)\right) .
$$

Being $x=0$ generic, and as $r \rightarrow 0^{+}$, we have

$$
\frac{1}{\omega_{k} r^{k}} \int_{B_{r}} H_{2} d\left\|V_{2}\right\| \longrightarrow \theta_{0} H_{2}(0)
$$

and

$$
-\frac{1}{\omega_{k} r^{k}} \int_{\partial B_{r} \cap M_{1}} \eta_{2} d \sigma_{2}=\frac{1}{\omega_{k} r^{k}} \int_{B_{r}} H_{1} d\left\|V_{1}\right\|+\mathrm{o}(1) \longrightarrow \theta_{0} H_{1}(0),
$$

thus, in view of (3.8), it remains to prove that $\int_{\partial B_{r} \cap \widetilde{\boldsymbol{M}}_{2}} \eta_{2} d \sigma_{2}=o\left(r^{k}\right)$ - at least for a suitable sequence of radii - to get the locality property at $x=0$, i.e. that $H_{1}(0)=$ $\mathrm{H}_{2}(0)$.

For every $X \in \mathrm{C}_{\mathrm{c}}^{1}\left(A, \mathbb{R}^{n}\right)$, we observe that, by the definition of the first variation, and thanks to the inclusion $M_{1} \cap A \subset M_{2}$,

$$
\begin{aligned}
\delta V_{2}(X) & =\int_{\boldsymbol{M}_{2}} \operatorname{div}_{\boldsymbol{M}_{2}} X \theta_{2} d \mathscr{H}^{k} \\
& =\int_{\widetilde{M}_{2}} \operatorname{div}_{\widetilde{M}_{2}} X \theta_{2} d \mathscr{H}^{k}+\int_{M_{1}} \operatorname{div}_{M_{1}} X \theta_{1} d \mathscr{H}^{k} \\
& =\delta \widetilde{V}_{2}(X)+\delta V_{1}(X),
\end{aligned}
$$

hence

$$
\left\|\delta \widetilde{V}_{2}\right\|(A) \leq\left\|\delta V_{1}\right\|(A)+\left\|\delta V_{2}\right\|(A) .
$$

Therefore, $\widetilde{V}_{2}$ has locally bounded first variation in $A$, like $V_{1}$ and $V_{2}$. Furthermore, using the genericity of 0 , one gets

$$
\frac{\left\|\delta V_{1}\right\|\left(B_{r}\right)}{\omega_{k} r^{k}} \rightarrow \theta_{0}\left|H_{1}(0)\right|
$$


and

$$
\frac{\left\|\delta V_{2}\right\|\left(B_{r}\right)}{\omega_{k} r^{k}} \rightarrow \theta_{0}\left|H_{2}(0)\right|
$$

as $r \rightarrow 0$, whence

$$
\left\|\delta V_{1}\right\|\left(B_{r}\right)+\left\|\delta V_{2}\right\|\left(B_{r}\right)=\mathrm{O}\left(r^{k}\right)
$$

and finally

$$
\left\|\delta \widetilde{V}_{2}\right\|\left(B_{r}\right)=\mathrm{O}\left(r^{k}\right)
$$

Let $g(r):=\left\|\widetilde{V}_{2}\right\|\left(B_{r}\right)$. Since $g(0)=0$ and $g$ is non-decreasing on $[0,+\infty)-$ thus $g$ has locally bounded variation - it holds for every $0 \leq \alpha<\beta<r_{0}$

$$
g(\beta)-g(\alpha)=\int_{\alpha}^{\beta} g^{\prime}(t) d t+\left|D^{s} g\right|((\alpha, \beta])
$$

where $g^{\prime}(t) d t$ and $D^{s} g$ are, respectively, the absolutely continuous part and the singular part of the distributional derivative $D g$. Besides, the coarea formula (1.5) yields

$$
\begin{aligned}
g(\beta)-g(\alpha) & =\int_{B_{\beta} \backslash B_{\alpha} \cap \widetilde{M}_{2}} d\left\|V_{2}\right\| \geq \int_{B_{\beta} \backslash B_{\alpha} \cap \widetilde{M}_{2}} \frac{\mid x_{M_{2} \mid}}{|x|} d\left\|V_{2}\right\| \\
& =\int_{\alpha}^{\beta} \int_{\partial B_{t} \cap \widetilde{M}_{2}} d \sigma_{2} d t .
\end{aligned}
$$

Since $D^{S} g$ and $g^{\prime}(t) d t$ are mutually singular, it follows that

$$
\int_{\alpha}^{\beta} g^{\prime}(t) d t \geq \int_{\alpha}^{\beta} \int_{\partial B_{t} \cap \widetilde{M}_{2}} d \sigma_{2} d t
$$

for almost every $0 \leq \alpha, \beta<r_{0}$. Therefore, by the Radon-Nikodym theorem,

$$
g^{\prime}(r) \geq \sigma_{2}\left(\partial B_{r} \cap \widetilde{M}_{2}\right), \quad \text { for a.e. } 0<r<r_{0} .
$$

We deduce that for almost every $r \in\left(0, r_{0}\right)$

$$
\left|\int_{\partial B_{r} \cap \widetilde{M}_{2}} \eta_{2} d \sigma_{2}\right| \leq \sigma_{2}\left(\partial B_{r} \cap \widetilde{M}_{2}\right) \leq g^{\prime}(r) .
$$

Then, it follows from the relative isoperimetric inequality (3.2) that for almost every $0<r<r_{0}$

$$
g(r)^{\frac{k-1}{k}} \leq C\left(\left\|\delta \widetilde{V}_{2}\right\|\left(B_{r}(x)\right)+g^{\prime}(r)\right),
$$

thus, by (3.11), for another suitable constant still denoted by $C$,

$$
g(r)^{\frac{k-1}{k}} \leq C\left(r^{k}+g^{\prime}(r)\right) .
$$


At the same time, the genericity of $x=0$ and the assumption $\Theta^{k}\left(\mathscr{H}^{k}\left\llcorner\widetilde{M}_{2}, x\right)=0\right.$ give

$$
g(r)=\mathrm{o}\left(r^{k}\right)
$$

Let $N$ be the set of real numbers in $\left(0, r_{0}\right)$ such that (3.12) and (3.13) hold. Clearly, $N$ has full measure in $\left(0, r_{0}\right)$. To conclude, we need to show that there exists a sequence of radii $\left(r_{h}\right)_{h \in \mathbb{N}} \in N$ decreasing to 0 , such that

$$
g^{\prime}\left(r_{h}\right)=\mathrm{o}\left(r_{h}^{k}\right)
$$

By contradiction, suppose that there exist a constant $C_{1}>0$ and a radius $0<r_{1}<$ $r_{0}$, such that $g^{\prime}(r) \geq C_{1} r^{k}$ for every $r \in N \cap\left(0, r_{1}\right)$. Then, by (3.13) and for an appropriate constant $C_{2}>0$,

$$
g(r)^{\frac{k-1}{k}} \leq C_{2} g^{\prime}(r)
$$

thus, for a.e. $0<r<r_{1}$,

$$
g(r)^{\frac{1-k}{k}} g^{\prime}(r) \geq \frac{1}{C_{2}} .
$$

Observing that $g(r)$ is non-decreasing and $g(0)=0$, we can integrate both sides of the inequality between 0 and $r$, to obtain

$$
k g(r)^{\frac{1}{k}} \geq \frac{r}{C_{2}},
$$

i.e. $g(r) \geq \frac{r^{k}}{\left(C_{2} k\right)^{k}}$, in contradiction with the fact that $g(r)=\mathrm{o}\left(r^{k}\right)$. In conclusion, by (3.12) and (3.15), there exists a sequence of radii $\left(r_{h}\right)_{h \in \mathbb{N}}$ decreasing to 0 such that (3.14) holds and

$$
\int_{\partial B_{r_{h}} \cap \widetilde{M}_{2}} \eta_{2} d \sigma_{2}=o\left(r_{h}^{k}\right) .
$$

Combining with (3.8), (3.9) and (3.10), we conclude the proof.

Corollary 3.5. Let $V_{M}=\mathbf{v}\left(M, \theta_{M}\right)$, be a rectifiable $k$-varifold with positive density and locally bounded first variation, such that

(i) there exist an open set $A \subset \mathbb{R}^{n}$ and a $\mathrm{C}^{2} k$-manifold $S$ such that $S \cap A \subset M$,

(ii) $\theta_{M}(x) \equiv \theta_{0}$ constant for $\mathscr{H}^{k}$-a.e. $x \in S \cap A$.

Then, $H_{M}(x)=H_{S}(x)$ for $\mathscr{H}^{k}$-almost every $x \in S \cap A$, where $H_{M}$ and $H_{S}$ denote, respectively, the generalized mean curvature of $V_{M}$ and the classical mean curvature of $S$.

Proof. It is an obvious consequence of the previous theorem by simply observing that, thanks to the divergence theorem for smooth sets, the classical mean curvature $H_{S}$ of $S$ coincides with the mean curvature of the varifold $\mathbf{v}\left(S, \theta_{0}\right)$. 
Conjecture 3.6. We would expect that the locality property of the mean curvature of $k$-varifolds, $k>1$, holds true under the sole hypothesis of locally bounded first variation. However, we have not been able to prove this assertion in full generality.

\section{Applications}

\subsection{Lower semicontinuity of the elastica energy for curves in $\mathbb{R}^{n}$}

Let $E$ be an open subset of $\mathbb{R}^{2}$ with smooth boundary $\partial E$ and let us consider the functional

$$
\mathcal{F}(E)=\int_{\partial E}\left(\alpha+\beta\left|\kappa_{\partial E}(y)\right|^{p}\right) d \mathscr{H}^{1}(y),
$$

where $p \geq 1, \kappa_{\partial E}(y)$ denotes the curvature at $y \in \partial E$ and $\alpha, \beta$ are positive constants. This functional is an extension to boundaries of smooth sets and to different curvature exponents of the celebrated elastica energy

$$
\int_{\gamma}\left(\alpha+\beta \kappa^{2}\right) d \mathscr{H}^{1}
$$

that was proposed in 1744 by Euler to study the equilibrium configurations of a thin, flexible beam $\gamma$ subjected to end forces. This energy, mainly used in elasticity theory, has also appeared to be of interest for a shape completion model in computer vision $[15,16]$.

Let $\overline{\mathcal{F}}$ denote the lower semicontinuous envelope - the relaxation - of $\mathcal{F}$ with respect to $\mathrm{L}^{1}$ convergence, i.e. for any measurable bounded subset $E \subset \mathbb{R}^{2}$,

$$
\overline{\mathcal{F}}(E)=\inf \left\{\liminf _{h \rightarrow \infty} \mathcal{F}\left(E_{h}\right), E_{h} \subset \mathbb{R}^{2} \text { open, } \partial E_{h} \in \mathrm{C}^{2},\left|E_{h} \Delta E\right| \rightarrow 0\right\},
$$

where $\left|E_{h} \Delta E\right|$ denotes the Lebesgue 2-dimensional outer measure of the symmetric difference of the sets $E_{h}$ and $E$.

Many properties of $\mathcal{F}$ and $\overline{\mathcal{F}}$ have been carefully studied in $[6,7,8]$. In particular, it has been proved in [6] that, whenever $E,\left(E_{h}\right)_{h} \subset \mathbb{R}^{2}, \partial E,\left(\partial E_{h}\right)_{h} \in \mathrm{C}^{2}$ and $\left|E_{h} \Delta E\right| \rightarrow 0$ as $h \rightarrow 0$, then

$$
\int_{\partial E}\left(\alpha+\beta\left|\kappa_{\partial E}\right|^{p}\right) d \mathscr{H}^{1} \leq \liminf _{h \rightarrow \infty} \int_{\partial E_{h}}\left(\alpha+\beta\left|\kappa_{\partial E_{h}}\right|^{p}\right) d \mathscr{H}^{1} \quad \text { for any } p>1 .
$$

This lower semicontinuity result is proved through a parameterization procedure that can be extended to the case of sets whose boundaries can be decomposed as a union of non crossing $\mathrm{W}^{2, p}$ curves. As a consequence, $\mathcal{F}(E)=\overline{\mathcal{F}}(E)$ for any $E$ in this class [6].

Thanks to Theorem 2.1, we can easily prove the lower semicontinuity of the $p$ elastica energy for curves in $\mathbb{R}^{n}, n \geq 2$, and for $p \geq 1$, thus getting an affirmative answer also for the case $p=1$. In this context, it is more appropriate to use the 
convergence in the sense of currents (see $[19,10]$ for the definitions and properties of currents), and the following result ensues:

Theorem 4.1. Let $\left(\boldsymbol{C}_{k}\right)_{k \in \mathbb{N}}$ with $\boldsymbol{C}_{k}=\bigcup_{i \in I(k)} C_{k, i}$ be a sequence of countable collections of disjoint, closed and uniformly bounded $\mathrm{C}^{2}$ curves in $\mathbb{R}^{n}$, converging in the sense of currents to a countable collection of disjoint, closed $\mathrm{C}^{2}$ curves $\varphi=\bigcup_{i \in I} C_{i}$, and satisfying

$$
\sup _{k \in \mathbb{N}} \sum_{i \in I(k)} \int_{C_{k, i}}\left(1+\left|\kappa_{C_{k, i}}\right|^{p}\right) d \mathscr{H}^{1}<+\infty .
$$

Then, for $\alpha, \beta \geq 0$,

$$
\sum_{i \in I} \int_{C_{i}}\left(\alpha+\beta\left|\kappa_{C_{i}}\right|^{p}\right) d \mathscr{H}^{1} \leq \liminf _{k \rightarrow \infty} \sum_{i \in I(k)} \int_{C_{k, i}}\left(\alpha+\beta\left|\kappa_{C_{k, i}}\right|^{p}\right) d \mathscr{H}^{1}
$$

for every $p \geq 1$.

Proof. With the notations of Section 1, we consider the sequence of varifolds $V_{k}=$ $\mathbf{v}\left(\varphi_{k}, 1\right)$. As an obvious consequence of our assumptions, the $V_{k}$ 's have uniformly bounded first variation and their curvatures are in $\mathrm{L}^{p}\left(\left\|V_{k}\right\|\right)$. By Allard's compactness theorem for rectifiable varifolds $[1,19]$, and possibly taking a subsequence, we get that $\left(V_{k}\right)$ converges in the sense of varifolds to an integral varifold $V$ with locally bounded first variation. In addition, by Theorem 2.34 and Example 2.36 in [3]

(i) if $p>1$ then the absolute continuity of $\delta V_{k}$ with respect to $\left\|V_{k}\right\|$ passes to the limit, i.e. $V$ has curvature in $\mathrm{L}^{p}$, and

$$
\int_{\mathbb{R}^{n}}\left(\alpha+\beta\left|\kappa_{V}\right|^{p}\right) d\|V\| \leq \liminf _{k \rightarrow \infty} \sum_{i \in I(k)} \int_{C_{k, i}}\left(\alpha+\beta\left|\kappa_{C_{k, i}}\right|^{p}\right) d \mathscr{H}^{1}
$$

(ii) if $p=1$, then $\delta V$ may not be absolutely continuous with respect to $V$, but the lower semicontinuity of both measures $\|\delta V\|$ and $\|V\|$ implies that

$$
\begin{aligned}
\int_{\mathbb{R}^{n}}\left(\alpha+\beta\left|\kappa_{V}\right|\right) d\|V\| & \leq \alpha\|V\|\left(\mathbb{R}^{n}\right)+\beta\|\delta V\|\left(\mathbb{R}^{n}\right) \\
& \leq \liminf _{k \rightarrow \infty} \sum_{i \in I(k)} \int_{C_{k, i}}\left(\alpha+\beta\left|\kappa_{C_{k, i}}\right|\right) d \mathscr{H}^{1} .
\end{aligned}
$$

Besides, as the convergence of the curves holds in the sense of currents, we know that $\mathscr{H}^{1}\left\llcorner\mathcal{C}=\left\|V_{\ell}\right\| \leq\|V\|\right.$, where $V_{\boldsymbol{e}}=\mathbf{v}(\boldsymbol{C}, 1)$. Since both $V_{\boldsymbol{C}}$ and $V$ have locally bounded first variation, it is a consequence of Theorem 2.1 that the curvatures of $V e$ 
and $V$ coincide $\mathscr{H}^{1}$-almost everywhere on $\mathcal{C}$. In conclusion, for every $p \geq 1$,

$$
\begin{aligned}
\sum_{i \in I} \int_{C_{i}}\left(\alpha+\beta\left|\kappa C_{i}\right|^{p}\right) d \mathscr{H}^{1} & \leq \int_{\mathbb{R}^{n}}\left(\alpha+\beta|\kappa V|^{p}\right) d\|V\| \\
& \leq \liminf _{k \rightarrow \infty} \sum_{i \in I(k)} \int_{C_{k, i}}\left(\alpha+\beta\left|\kappa_{C_{k, i}}\right|^{p}\right) d \mathscr{H}^{1}
\end{aligned}
$$

and the theorem ensues.

Remark 4.2. Using the same kind of arguments, the result can be extended to unions of $\mathrm{W}^{2, p}$ curves in $\mathbb{R}^{n}, p \geq 1$.

Remark 4.3. In higher dimension, the elastica energy becomes the celebrated Willmore energy [20], that can also be generalized to arbitrary mean curvature exponent under the form

$$
\int_{S}\left(\alpha+\beta\left|H_{S}\right|^{p}\right) d \mathscr{H}^{k}
$$

with $S$ a smooth $k$-surface in $\mathbb{R}^{n}$ and $H_{S}$ its mean curvature vector. Our partial locality result for rectifiable $k$-varifolds in $\mathbb{R}^{n}$ is not sufficient to prove the extension to smooth $k$-surfaces of the semicontinuity result for curves stated above. This is due to the fact that the limit varifold obtained in the proof of Theorem 4.1 might not have a locally constant multiplicity. For instance, consider the varifold $\hat{V}$ obtained by adding the horizontal $x$-axis (with multiplicity 1 ) to the varifold $V$ that we have built in section 2.2. Then, one immediately observes that the $x$-axis is contained in the support of $\|\hat{V}\|$, but the multiplicity $\hat{\theta}$ of $\hat{V}$ is not locally constant at the points corresponding to the "fat" Cantor set ( $\hat{\theta}$ takes both values 1 and 2 in any neighbourhood of such points). Therefore, Theorem 3.4 cannot be directly used in this situation.

Were the locality property true in general, one would obtain the lower semicontinuity result in any dimension $k$ and codimension $n-k$, and for any $p \geq 1$. Currently, to our best knowledge, the most general lower semicontinuity result for the case $k>1$ is due to R. Schätzle [18, Thm 5.1] and is valid when $p \geq 2$.

\subsection{Relaxation of functionals for image reconstruction}

Recall that for any smooth function $u: \mathbb{R}^{n} \rightarrow \mathbb{R}$ and for almost every $t \in \mathbb{R}, \partial\{u \geq t\}$ is a union of smooth hypersurfaces whose mean curvature at a point $x$ is given by

$$
H(x)=\operatorname{div} \frac{\nabla u}{|\nabla u|}(x) .
$$

Thus, for any open set $\Omega \subset \mathbb{R}^{n}$ and by application of the coarea formula, we get

$$
\int_{-\infty}^{+\infty} \int_{\Omega \cap \partial\{u \geq t\}}\left(1+\left|H_{\partial\{u \geq t\}}\right|^{p}\right) d \mathscr{H}^{n-1} d t=\int_{\Omega}|\nabla u|\left(1+\left|\operatorname{div} \frac{\nabla u}{|\nabla u|}\right|^{p}\right) d x,
$$


where the integrand of the right term is taken to be zero whenever $|\nabla u|=0$. The minimization of the energy

$$
\mathcal{F}(u):=\int_{\Omega}|\nabla u|\left(1+\left|\operatorname{div} \frac{\nabla u}{|\nabla u|}\right|^{p}\right) d x
$$

has been proposed in the context of digital image processing [13, 12, 14] as a variational criterion for the restoration of missing parts in an image. It is therefore natural to study the connections between $\mathscr{F}(u)$, and its relaxation $\widetilde{\mathcal{F}}(u)$ with respect to the convergence of functions in $\mathrm{L}^{1}$. In particular, the question whether $\mathcal{F}(u)=\overline{\mathcal{F}}(u)$ for smooth functions has been addressed in [4] and a positive answer has been given whenever $n \geq 2$ and $p>n-1$. Following the same proof line combined with our Theorem 4.1 and with Schätzle's Theorem 5.1 in [18], one can prove the following:

Theorem 4.4. Let $u \in \mathrm{C}^{2}\left(\mathbb{R}^{n}\right)$. Then

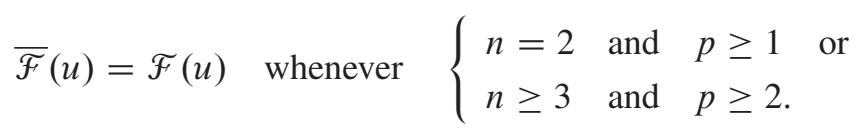

Proof. Let $\left(u_{h}\right)_{h \in \mathbb{N}} \subset L^{1}\left(\mathbb{R}^{n}\right) \cap C^{2}\left(\mathbb{R}^{n}\right)$ converge to $u$ in $L^{1}\left(\mathbb{R}^{n}\right)$ and set $L:=$ $\liminf _{h \rightarrow \infty} \mathcal{F}\left(u_{h}\right)$, assuming with no loss of generality that $L<\infty$. Using Cavalieri's formula and possibly taking a subsequence, it follows that for almost every $t \in \mathbb{R}$,

$$
\mathbb{1}_{\left\{u_{h} \geq t\right\}} \rightarrow \mathbb{1}_{\{u \geq t\}} \quad \text { in } L^{1}\left(\mathbb{R}^{n}\right) .
$$

Observing that, by Sard's lemma, $\left\{u_{h} \geq t\right\}, h \in \mathbb{N}$, and $\{u \geq t\}$ have smooth boundaries for almost every $t \in \mathbb{R}$, we get that $\partial\left\{u_{h} \geq t\right\}$ converges to $\partial\{u \geq t\}$ in the sense of rectifiable currents for almost every $t \in \mathbb{R}$ [19]. Therefore, applying either Theorem 4.1 or Theorem 5.1 in [18], we obtain that for almost every $t \in \mathbb{R}$

$$
\begin{array}{r}
\int_{\partial\{u \geq t\}}\left(1+\left|H_{\{u \geq t\}}\right|^{p}\right) d \mathscr{H}^{n-1} \leq \liminf _{h \rightarrow \infty} \int_{\partial\left\{u_{h} \geq t\right\}}\left(1+\left|H_{\left\{u_{h} \geq t\right\}}\right|^{p}\right) d \mathscr{H}^{n-1} \\
\text { whenever } \begin{cases}n=2 & \text { and } p \geq 1 \text { or } \\
n \geq 3 & \text { and } p \geq 2\end{cases}
\end{array}
$$

Integrating over $\mathbb{R}$ and using Fatou's lemma, we get

$$
\mathcal{F}(u) \leq \liminf _{h \rightarrow \infty} \mathcal{F}\left(u_{h}\right),
$$

thus $\mathcal{F}$ is lower semicontinuous in the class of $C^{2}$ functions and coincides with $\overline{\mathcal{F}}$ on that class.

Acknowledgments. We warmly thank Fabrice Bethuel for his constant support and Luca Mugnai for useful comments on the first version of the paper. 


\section{References}

[1] W. K. Allard, On the first variation of a varifold, Ann. of Math. (2) 95 (1972), pp. $417-$ 491.

[2] F. J. Almgren, The theory of varifolds, Mimeographed notes, Princeton Univ. Press, Princeton, NJ (1965).

[3] L. Ambrosio, N. Fusco and D. Pallara, Functions of bounded variation and free discontinuity problems, Oxford Mathematical Monographs, The Clarendon Press Oxford University Press, New York, 2000.

[4] L. Ambrosio and S. Masnou, A direct variational approach to a problem arising in image reconstruction, Interfaces Free Bound. 5 (2003), pp. 63-81.

[5] G. Anzellotti and R. Serapioni, $C^{k}$-rectifiable sets, J. Reine Angew. Math. 453 (1994), pp. 1-20.

[6] G. Bellettini, G. Dal Maso and M. Paolini, Semicontinuity and relaxation properties of a curvature depending functional in 2D, Ann. Scuola Norm. Sup. Pisa Cl. Sci. (4) 20 (1993), pp. 247-297.

[7] G. Bellettini and L. Mugnai, Characterization and representation of the lower semicontinuous envelope of the elastica functional, Ann. Inst. H. Poincaré Anal. Non Linéaire 21 (2004), pp. 839-880.

[8] _ A varifolds representation of the relaxed elastica functional, J. Convex Anal. 14 (2007), pp. 543-564.

[9] K. A. Brakke, The motion of a surface by its mean curvature, Mathematical Notes 20, Princeton University Press, Princeton, N.J., 1978.

[10] H. Federer, Geometric measure theory, Die Grundlehren der mathematischen Wissenschaften, Band 153, Springer-Verlag New York Inc., New York, 1969.

[11] T. Ilmanen, Elliptic regularization and partial regularity for motion by mean curvature, Mem. Amer. Math. Soc. 108 (1994), no. 520.

[12] S. Masnou, Disocclusion: a variational approach using level lines, IEEE Trans. Image Process. 11 (2002), pp. 68-76.

[13] S. Masnou and J.-M. Morel, Level lines based disocclusion, in: Proc. ICIP'98 IEEE Int. Conf. on Image Processing, 3, pp. 259-263, Chicago, USA, 1998.

[14] , On a variational theory of image amodal completion, Rend. Sem. Mat. Univ. Padova 116 (2006), pp. 211-252.

[15] D. Mumford, Elastica and computer vision, Algebraic geometry and its applications (West Lafayette, IN, 1990), Springer, New York, 1994, pp. 491-506.

[16] M. Nitzberg and D. Mumford, The 2.1-D Sketch, in: Proc. 3rd Int. Conf. on Computer Vision, pp. 138-144, Osaka, Japan, 1990.

[17] R. Schätzle, Quadratic tilt-excess decay and strong maximum principle for varifolds, Ann. Sc. Norm. Super. Pisa Cl. Sci. (5) 3 (2004), pp. 171-231. 
[18] Lower semicontinuity of the Willmore functional for currents, to appear in J. Diff. Geom. (2008).

[19] L. Simon, Lectures on Geometric Measure Theory, vol. 3, Proc. Centre for Math. Anal., 1983.

[20] T. J. Willmore, Note on embedded surfaces, An. Şti. Univ. "Al. I. Cuza” Iaşi Secţ. I a Mat. (N.S.) 11B (1965), pp. 493-496.

Received March 8, 2008; revised September 2, 2008.

\section{Author information}

Gian Paolo Leonardi, DMPA, Univ Modena, via Campi 213/b, 41100, Modena, Italy.

E-mail: gianpaolo.leonardi@unimore.it

Simon Masnou, UPMC Univ Paris 06, UMR 7598, Lab. J.-L. Lions, F-75005, Paris, France.

E-mail: masnou@ann.jussieu.fr 